\title{
Detection of aquaporin-4 antibody using aquaporin-4 extracellular loop-based carbon nanotube biosensor for the diagnosis of neuromyelitis optica
}

\author{
Manki Son ${ }^{\mathrm{a}}$, Daesan Kim ${ }^{\mathrm{b}}$, Kyung Seok Park ${ }^{\mathrm{c}}$, Seunghun Hong ${ }^{\mathrm{d}, *}$, Tai Hyun Park ${ }^{\mathrm{a}, \mathrm{e}, \mathrm{f}, * *}$ \\ a Interdisciplinary Program for Bioengineering, Seoul National University, Seoul 151-742, South Korea \\ ${ }^{\mathrm{b}}$ Department of Biophysics and Chemical Biology, Seoul National University, Seoul 151-747, South Korea

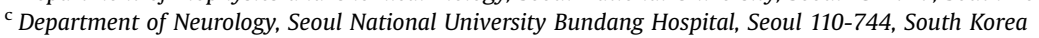 \\ ${ }^{\mathrm{d}}$ Department of Physics and Astronomy and Institute of Applied Physics, Seoul National University, Seoul 151-747, South Korea \\ e School of Chemical and Biological Engineering, Seoul National University, Seoul 151-742, South Korea \\ ${ }^{\mathrm{f}}$ Advanced Institutes of Convergence Technology, Suwon 433-270, South Korea
}

\section{A R T I C L E I N F O}

\section{Article history:}

Received 21 August 2015

Received in revised form 3 November 2015

Accepted 10 November 2015

Available online 12 November 2015

Keywords:

Neuromyelitis optica

Aquaporin-4

Carbon nanotube

Field-effect transistor

\begin{abstract}
A B S T R A C T
Here we propose a carbon nanotube (CNT) field-effect transistor (FET) functionalized with aquaporin-4 (AQP4) extracellular loop peptides for the rapid detection of AQP4 antibody without pretreatment. Neuromyelitis optica (NMO) is a rare disease of the central nerve system that affects the optic nerves and the spinal cord. NMO-IgG, a serum antibody in patients, is highly specific for NMO and targets AQP4. We synthesized AQP4 extracellular loop peptides, known as primary autoimmune target in NMO, and immobilized them onto CNT-FET. The sensor showed p-type FET characteristics after the functionalization of peptides. The sensor was able to detect antibody with a detection limit of $1 \mathrm{ng} \mathrm{l^{-1 }}$. Moreover, AQP4 antibody in human serum was detected without any pretreatment. These results indicate that the biosensor can be used for rapid and simple detection of NMO antibody.
\end{abstract}

(c) 2015 Elsevier B.V. All rights reserved.

\section{Introduction}

Neuromyelitis optica (NMO, also known as Devic's disease) is a severe and uncommon disease of the central nerve system (CNS) that principally attacks the optic nerves and spinal cords (Wingerchuk et al., 1999, 2006). The symptoms of the disease, including blindness and paralysis, are very serious. NMO is commonly confused with multiple sclerosis (MS) because the symptoms are similar. Since the treatments for the two diseases are different, an accurate diagnosis in the early phase is important. In 2004, NMOIgG was found to be a highly specific biomarker in human blood and enabled the differentiation of NMO and MS patients (Lennon et al., 2004, 2005). This disease's specific antibody targets aquaporin-4 (AQP4), a transmembrane protein expressed in the CNS, that regulates water transport in astrocytes and ependymal cells. Various laboratory tests using AQP4 were developed for the detection of AQP4 antibody with tissue-, cell- and protein-based assays (Fazio et al., 2009; Hayakawa et al., 2008; Jarius et al., 2010; Marnetto et al., 2009; McKeon et al., 2009; Paul et al., 2007;

\footnotetext{
* Corresponding author.

** Corresponding author at: Interdisciplinary Program for Bioengineering, Seoul National University, Seoul 151-742, South Korea

E-mail addresses: seunghun@snu.ac.kr (S. Hong), thpark@snu.ac.kr (T.H. Park).
}

Takahashi et al., 2007; Waters et al., 2008). However, these methods have the limitations such as low sensitivity and long analytical time for diagnosis.

In a recent study, three extracellular loops (A, C and E) of AQP4 were found to contain disease-specific epitopes, which could be used in an assay for the diagnosis of NMO (Iorio et al., 2013; Pisani et al., 2011). In order to overcome the drawbacks of existing methods, we propose a peptide-based Carbon nanotube (CNT) biosensor for the rapid detection of AQP4 antibody in human serum. A CNT-based field-effect transistor (FET) has been used for the rapid detection of biological molecules with high sensitivity (Jin et al., 2012; Lim et al., 2013, 2014; Park et al., 2012, 2014; Song et al., 2014). CNTs have a large surface to volume ratio due to their unique one-dimensional nanoscale structures. Thus, the electrical properties of CNTs could be easily changed by adsorptions of biomolecules onto their surfaces (Allen et al., 2007). We synthesized AQP4 extracellular loop peptides and directly immobilized them onto CNT channels. Using this biosensor, we could detect AQP4 antibody, a biomarker of NMO disease, with a detection limit of $1 \mathrm{ng} \mathrm{l^{-1 }}$. Furthermore, the sensor was able to detect the antibody without any pretreatment of the human serum. 


\section{Materials and methods}

\subsection{Synthesis of peptide}

Three kinds of AQP4 extracellular loop peptides (A: GGSENPLPVFFF, C: TPPSVVGGLGVTTVHGNLTAGFFF, E: GNWENHWFFF) with purity higher than 95\% were purchased from Peptron (Korea). All peptides were dissolved and diluted at $1 \mathrm{mg} \mathrm{ml}^{-1}$ with distilled water. The diluted peptide solution was stored at $-20^{\circ} \mathrm{C}$ and thawed before used.

\subsection{Fabrication of biosensor}

The CNT-FET was fabricated via the photolithography process as previously. Since a massive fabrication method provides the uniform characteristics of devices, CNT-FETs were fabricated via surface-programmed assembly method (Lee et al., 2006). Briefly, photoresist (PR) was used to fabricate patterns for the channel parts of FETs on a silicon oxide wafer. The region without patterns were dipped into octadecyltrichlorosilane (OTS) solution (OTS: Hexane $=1: 400$ ) for $7 \mathrm{~min}$. The wafer was rinsed using acetone to remove the PR patterns. The OTS-patterned water was incubated in swCNT solution $(0.05 \mathrm{mg} / \mathrm{ml}$ in dichlorobenzene) for $20 \mathrm{~s}$. CNTs were adsorbed specifically on the patterned $\mathrm{SiO}_{2}$ regions owing to self-assembled OTS molecules on the surface. After the CNT patterning process, $\mathrm{Ti} / \mathrm{Au}(10 / 30 \mathrm{~nm})$ electrodes were developed via thermal evaporation. Finally, the insulating layer was fabricated using PR (DNR) to prevent the electrodes from contacting the solution. To immobilize the peptides, $1 \mu \mathrm{l}$ of peptide solution was placed on the CNT channel of the fabricated CNT-FET for $4 \mathrm{~h}$. After immobilization, unbound peptides were washed 3 times with distilled water.

\subsection{Characterization of peptide immobilization}

The imaging of CNT channels were performed using AFM (Asylum Research, USA) under ambient conditions. At first, the pristine CNT channels were imaged in tapping mode with a scan rate of $0.2 \mathrm{~Hz}$. After the immobilization of the peptides, AFM imaging of the CNT channels was performed in the same mode at $0.1 \mathrm{~Hz}$ to avoid undesired noise.

\subsection{Electrical measurement}

For the electrical signal measurement, $20 \mu \mathrm{l}$ of PBS with $\mathrm{pH}$ 7.4 was added to the peptide-immobilized CNT channel. A $0.1 \mathrm{~V}$ bias voltage was applied between the source and drain electrodes, while the gate voltage was grounded. Current changes were monitored with a 2636A Dual-channel system source meter instrument (Keithley, USA) and a MST 8000 probestation (MS TECH, Korea).

\section{Results and discussion}

\subsection{Construction of peptide-based biosensor}

Three kinds of AQP4 extracellular loop peptides were synthesized, and three molecules of phenylalanine (Phe) were added to the C-terminus in order to immobilize the synthesized peptides onto CNTs via pi-pi interactions between CNTs and the aromatic rings of Phe (Chen et al., 2001; Poenitzsch et al., 2007). In order to have a maximal capacity for antibody sensing, sufficiently high concentrations of the peptides were immobilized on the CNT channels as a monolayer via self-assembly and unbounds were washed away with distilled water. The fabricated CNT biosensor

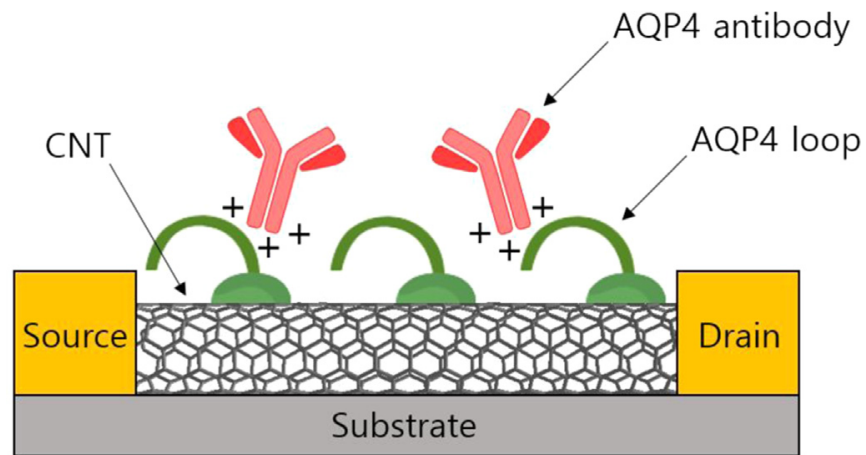

Fig. 1. Schematic diagram showing the sensing mechanism of an AQP4 loop peptide-based CNT-FET biosensor. Positively-charged AQP4 antibody binds with the AQP4 extracellular loop peptides on CNTs, which gives a positive field-effect on the FET and decreases its electric conductance.

could detect AQP4 antibody, which had a $\mathrm{pI}$ value of 9-10 and was positively-charged in PBS of pH 7.4, with a decreased conductance. Fig. 1 shows the sensing mechanism of the AQP4 loop peptidebased biosensor.

To confirm the immobilization of the peptides, an atomic force microscopy (AFM) image analysis was performed. Fig. 2a shows the AFM images of the CNT channels before and after the immobilization of three kinds of AQP4 extracellular loop peptides. When we compared the height profiles of specific CNT regions, the height was increased by $0.71 \pm 3.13 \mathrm{~nm}$ with AQP4 loop A, $0.56+2.9 \mathrm{~nm}$ with AQP4 loop C and $1.4+2.9 \mathrm{~nm}$ with AQP4 loop $\mathrm{E}$ (Fig. 2b). These results show a successful immobilization of the AQP4 extracellular loop peptides on CNT channels.

\subsection{Electrical characteristic of biosensor}

To evaluate the electrical property of the biosensor, the sourcedrain current-voltage $\left(I_{\mathrm{SD}}-V_{\mathrm{SD}}\right)$ of $\mathrm{CNT}$ channels were measured (Fig. 3a). Although the resistance of the FET sensor was increased from $6.55 \times 10^{5} \Omega$ to $1.54 \times 10^{6} \Omega$ with AQP4 loop A, $6.57 \times 10^{5} \Omega$ to $1.23 \times 10^{6} \Omega$ with AQP4 loop $C$ and $6.59 \times 10^{5} \Omega$ to $1.11 \times 10^{6} \Omega$ with AQP4 loop E, the linearity of the curve was maintained before and after the immobilization of the peptides. We constructed a liquid-ion gated FET system with PBS (pH 7.4). The source-drain current of the CNT channel was measured while increasing the gate voltage (Fig. 3b). The current between the source and the drain $\left(I_{\mathrm{SD}}\right)$ decreased with an increasing gate voltage $\left(V_{\mathrm{G}}\right)$, indicating p-type FET behavior. These electrical characteristics indicate that the sensor can detect positively-charged AQP4 antibody in PBS.

\subsection{Detection of AQP4 antibody using biosensor}

The sensing ability of this sensor was evaluated using antibodies in PBS solution. A PBS droplet of $20 \mu \mathrm{l}$ was added to the peptide-immobilized CNT channel, and the sample solution containing AQP4 antibody (ab46182, Abcam, UK) was injected. Fig. 4a shows the real-time conductance of the sensor with concentrations of AQP4 antibody ranging from $1 \mathrm{ng} \mathrm{l}^{-1}$ to $1 \mu \mathrm{g} \mathrm{l}^{-1}$. Each AQP4 extracellular loop peptide-based sensor showed a dose-dependent response. The sensor was able to detect AQP4 antibody at a concentration as low as $1 \mathrm{ng} \mathrm{l}^{-1}$ with AQP4 loop E. In our previous research for the detection of AQP4 antibody, nanovesiclebased CNT biosensor showed the detection limit of $10 \mathrm{ng} \mathrm{l}^{-1}$ antibody (Park et al., 2014). The LOD was improved by ten-fold due to the shortened distance between CNTs and antibody, since the small size peptides were used. Fig. 4b shows the normalized sensitivity of each peptide to different concentrations of AQP4 
a

\section{Pristine}

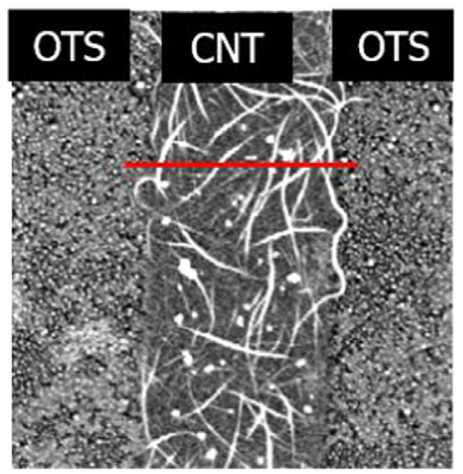

AQP4 loop A

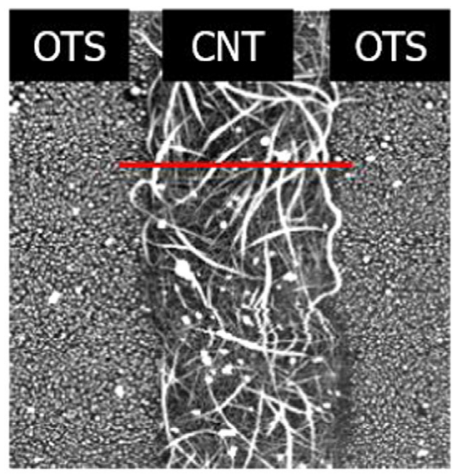

b

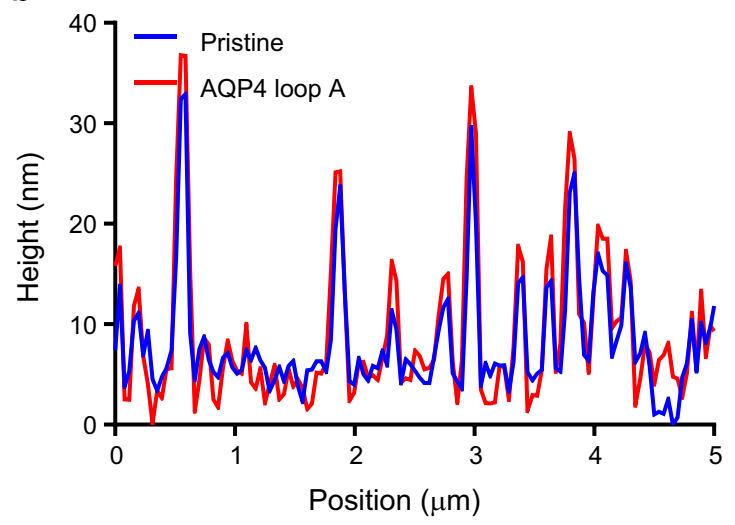

Pristine

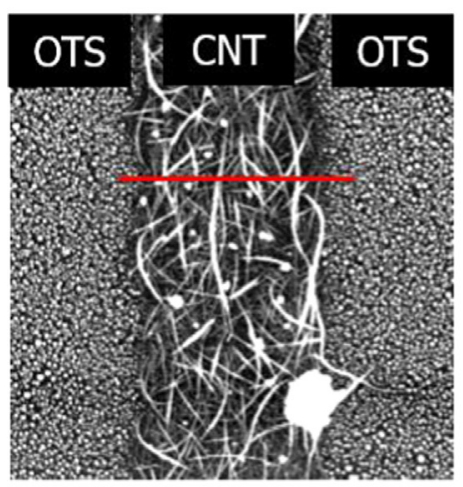

Pristine

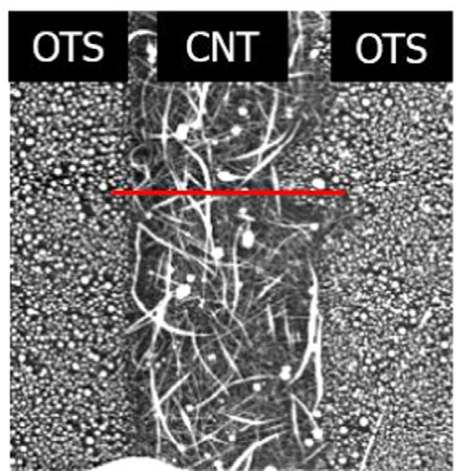

AQP4 loop C
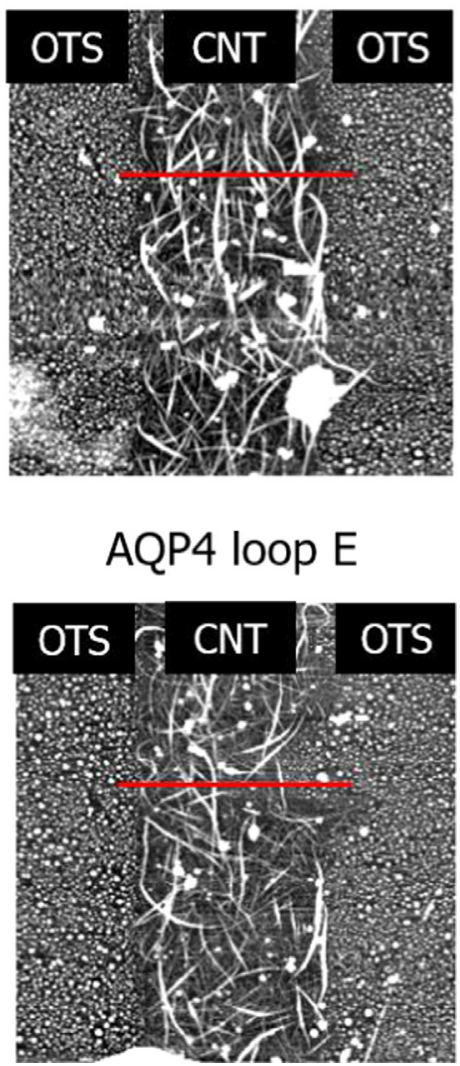
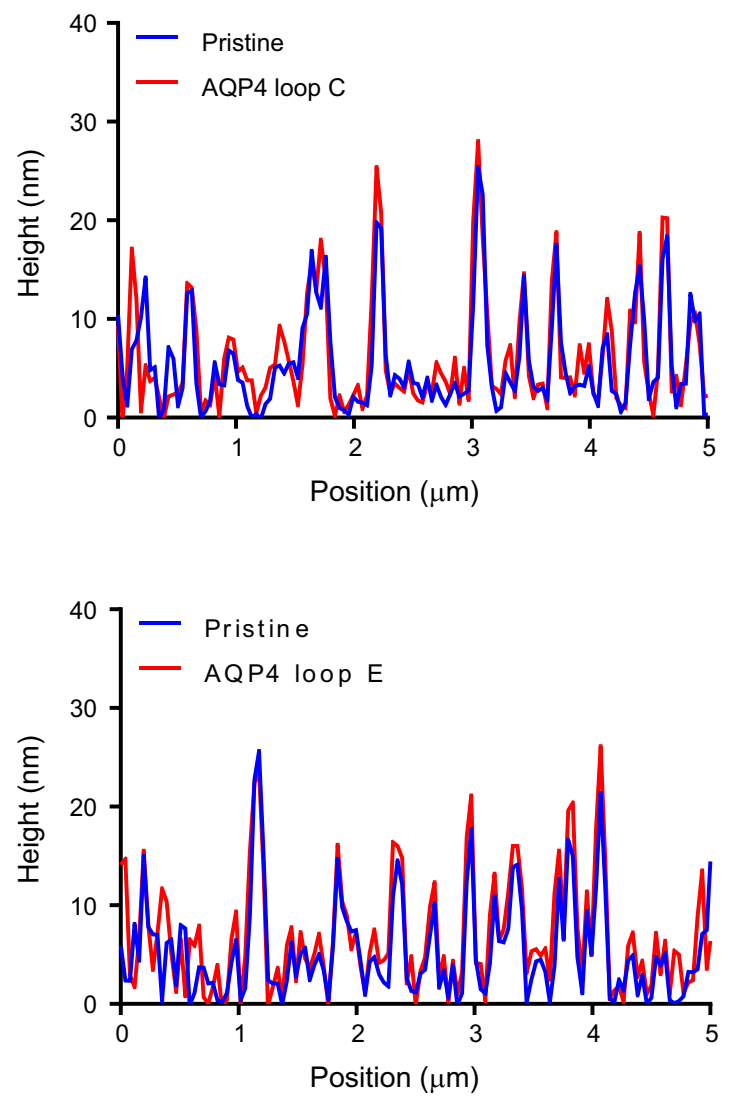

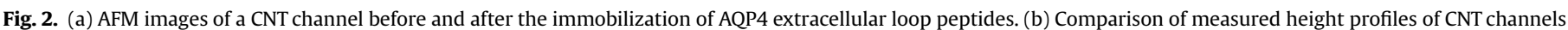

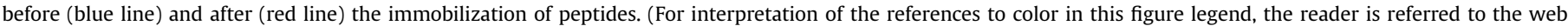
version of this article.)

antibody. AQP4 loop E showed the highest sensitivity to AQP4 antibody among the three peptides. This result was attributed to the different affinity of each peptide with the antibody and different lengths of the peptides on the CNT channel.

\subsection{Detection of AQP4 antibody in human serum using biosensor}

It was reported that the antibody level of NMO patients is $13.984 \pm 5.981 \mathrm{~g} \mathrm{l}^{-1}$ (Chen et al., 2014). To evaluate the usability of the sensor for the diagnosis of NMO, $1 \mathrm{~g} \mathrm{l}^{-1}$ of AQP4 antibody in human serum (H4522, SIGMA, US) was tested. Serum samples containing AQP4 antibody were prepared to $1: 10^{5}$ dilution in PBS to avoid a non-specific response (Lim et al., 2014). As shown in Fig. 5a, the sensor did not respond to the human serum without AQP4 antibody, but the conductance decreased when antibodies were present in the serum samples. The RSD values were $8.33 \%$ with AQP4 loop A, 23.08\% with AQP4 loop C and 2.17\% with AQP4 loop $\mathrm{E}(n=3)$ (Fig. $5 \mathrm{~b})$. These results indicate that the sensor can be effectively used for the rapid and easy diagnosis of NMO. This biosensor is for a single-use. It was difficult to recover the current signal after use, since the binding of antibody and peptide was not completely removed with washing process. However, mass 
a
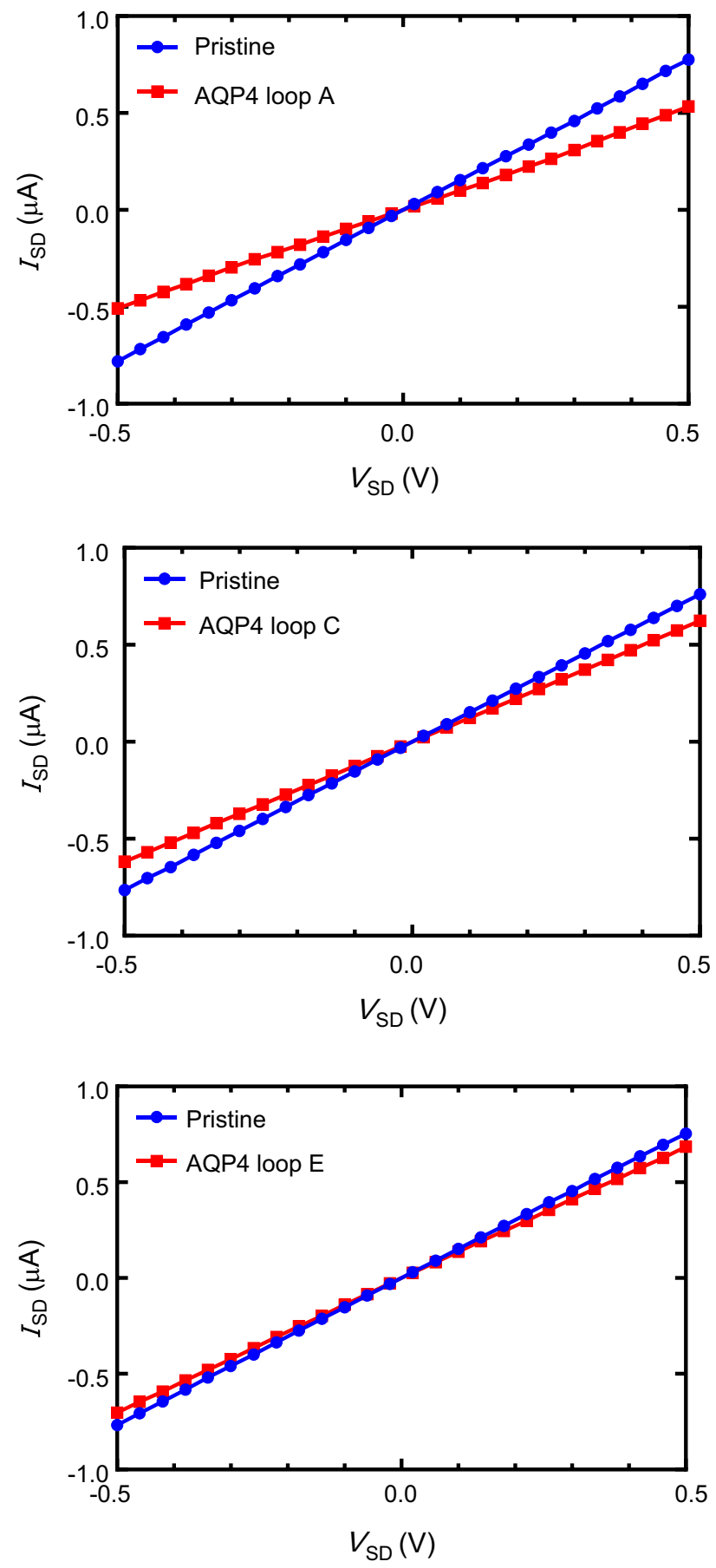

b
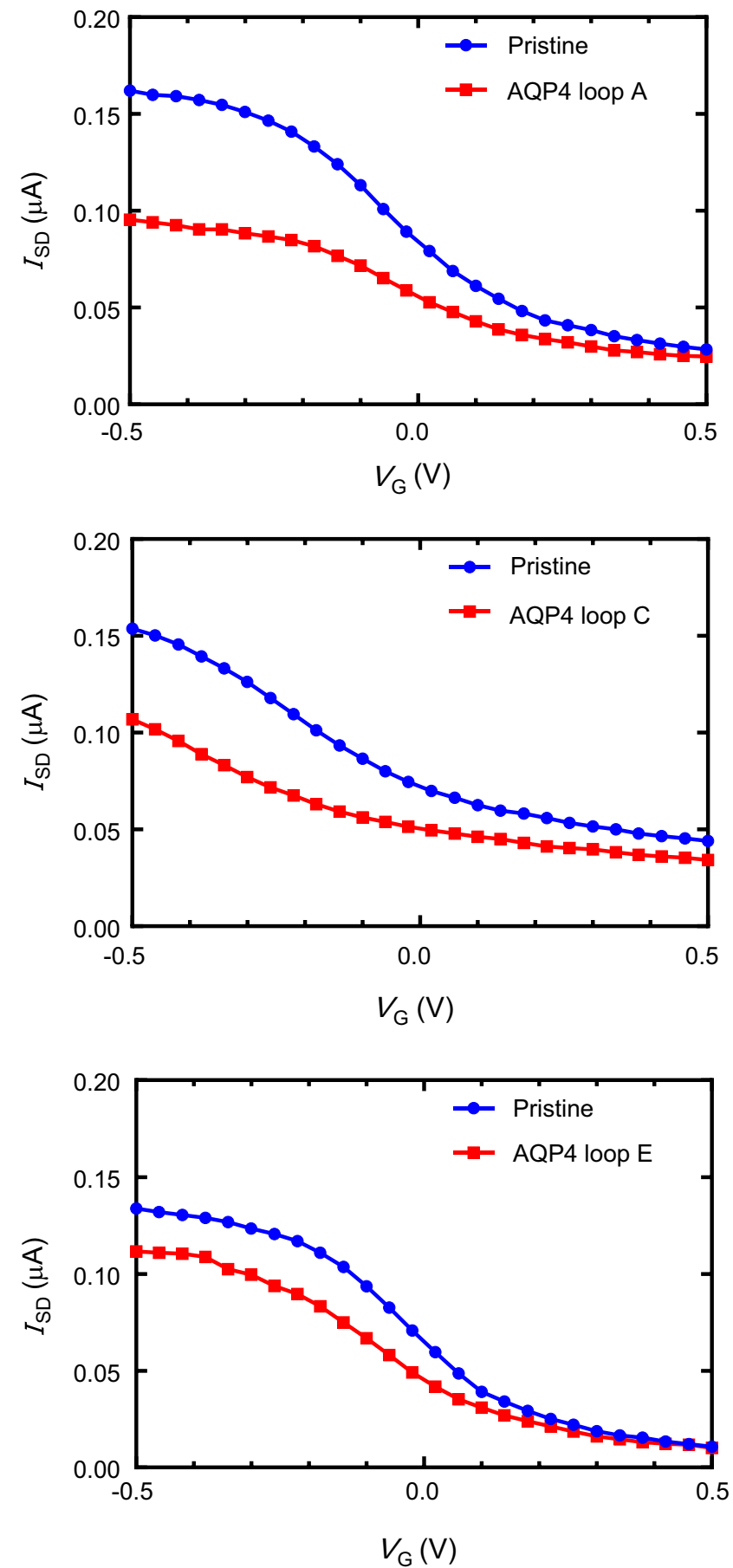

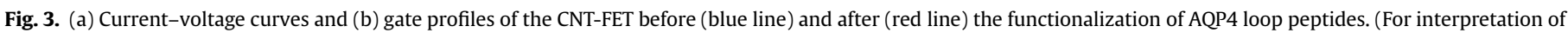
the references to color in this figure legend, the reader is referred to the web version of this article.)

production of CNT-based sensor is available with the lithography method and it requires only a small volume of peptide for the functionalization. It also has advantages in simple and fast detection of antibody without complex pretreatment procedures.

\section{Conclusions}

We developed a rapid sensor for the diagnosis of NMO using peptide-based CNT-FET. AQP4 extracellular loop peptides, which bind with the serum biomarker of NMO, were synthesized and three molecules of Phe were added to the C-terminal for immobilization on CNTs. The synthesized peptides were directly immobilized onto CNT channels in the FET sensor. The constructed biosensor showed p-type FET characteristics and could detect a positively charged antibody. The sensor can detect $1 \mathrm{ng} \mathrm{l}^{-1}$ of AQP4 antibody with the AQP4 loop E peptide. Moreover, human serum antibody was detected without any pretreatment. Due to its high sensitivity and rapidity, this biosensor can be effectively used as a simple and easy diagnostic method for NMO. 
a

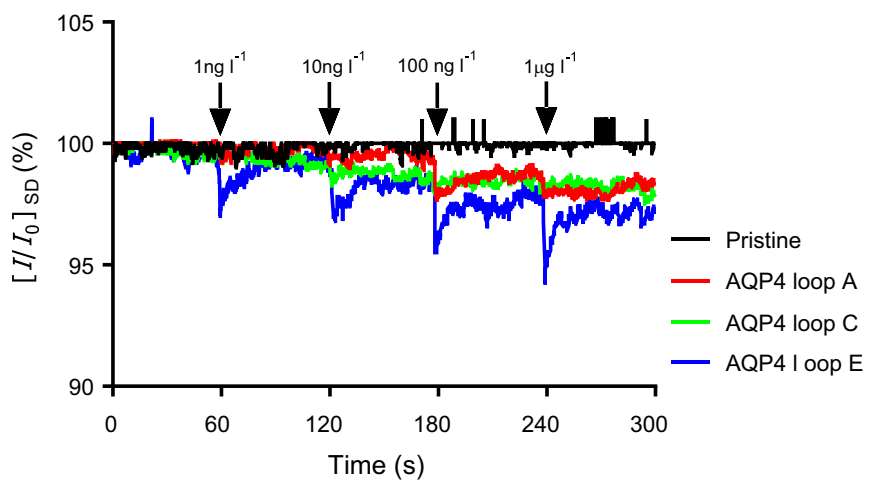

b

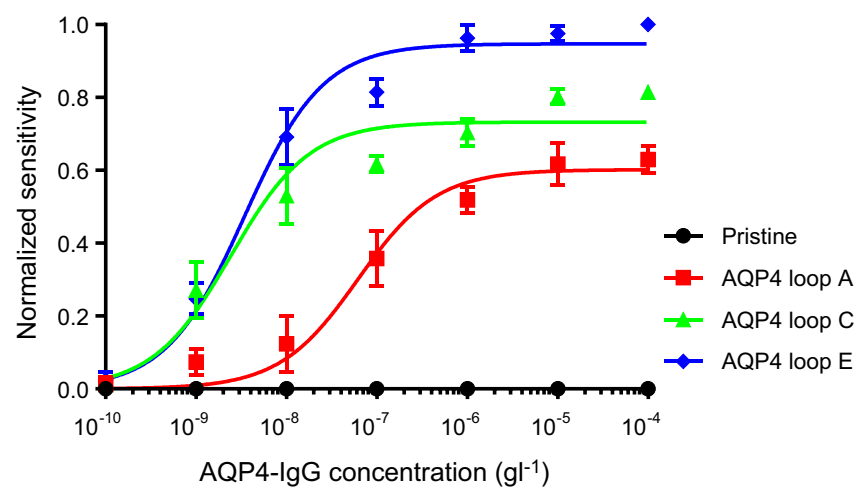

Fig. 4. (a) Real-time responses of AQP4 loop peptide-based CNT-FETs to AQP4 antibody at concentrations ranging from $1 \mathrm{ng} \mathrm{l}^{-1}$ to $1 \mu \mathrm{g} \mathrm{l}^{-1}$. (b) Normalized sensitivity of biosensors based on pristine CNTs (black line), CNTs functionalized with AQP4 loop A (red line), C (green line) and E (blue line) to antibody. Error bars, s.e.m. three replicates. (For interpretation of the references to color in this figure legend, the reader is referred to the web version of this article.)

\section{Acknowledgments}

This work was supported by the Seoul National University Research Grant. This study was also supported by a Grant from the National Research Foundation of Korea (NRF), funded by the Ministry of Science, ICT and Future Planning (Grant no. 2014053108). SH acknowledges the support from the NRF (No. 2013M3A6B2078961).

\section{References}

Allen, B.L., Kichambare, P.D., Star, A., 2007. Adv, Mater. 19, 1439-1451. Chen, R.J., Zhang, Y., Wang, D., Dai, H., 2001. J. Am. Chem. Soc. 123, 3838-3839. Chen, Y., Li, R., Wu, A.M., Lu, Z.Q., Hu, X.Q., 2014. Neurol. Sci. 2, 215-220. Fazio, R., Malosio, M.L., Lampasona, V., De Feo, D., Privitera, D., Marnetto, F., Centonze, D., Ghezzi, A., Comi, G., Furlan, R., Martino, G., 2009. Mult. Scler. 15, $1153-1163$.

Hayakawa, S., Mori, M., Okuta, A., Kamegawa, A., Fujiyoshi, Y., Yoshiyama, Y., Mitsuoka, K., Ishibashi, K., Sasaki, S., Hattori, T., Kuwabara, S., 2008. J. Neuroimmunol. 196, 181-187.

Iorio, R., Fryer, J.P., Hinson, S.R., Fallier-Becker, P., Wolburg, H., Pittock, S.J., Lennon, V.A., 2013. J. Autoimmun. 40, 21-27.

Jarius, S., Probst, C., Borowski, K., Franciotta, D., Wildemann, B., Stoecker, W. Wandinger, K.P., 2010. J. Neurol. Sci. 291, 52-56.

Jin, H.J., Lee, S.H., Kim, T.H., Park, J., Song, H.S., Park, T.H., Hong, S., 2012. Biosens. Bioelectron. 35, 335-341.

Lee, M., Im, J., Lee, B.Y., Myung, S., Kang, J., Huang, L., Kwon, Y.K., Hong, S., 2006. Nat. Nanotechnol. 1, 66-71.

Lennon, V.A., Wingerchuk, D.M., Kryzer, T.J., Pittock, S.J., Lucchinetti, C.F., Fujihara, K., Nakashima, I., Weinshenker, B.G., 2004. Lancet 364, 2106-2112.

Lennon, V.A., Kryzer, T.J., Pittock, S.J., Verkman, A.S., Hinson, S.R., 2005. J. Exp. Med 202, 473-477.

Lim, J.H., Park, J., Ahn, J.H., Jin, H.J., Hong, S., Park, T.H., 2013. Biosens. Bioelectron. 39, 244-249. a

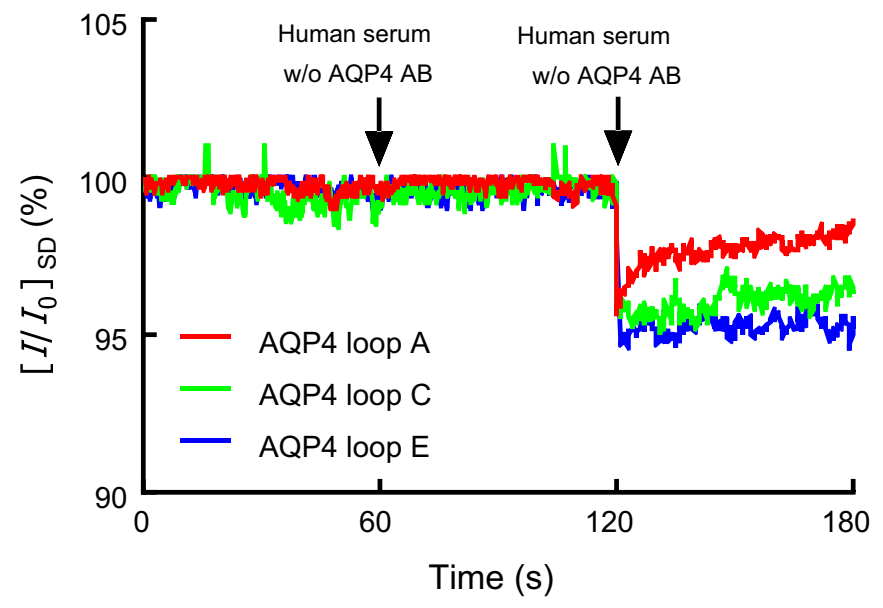

b

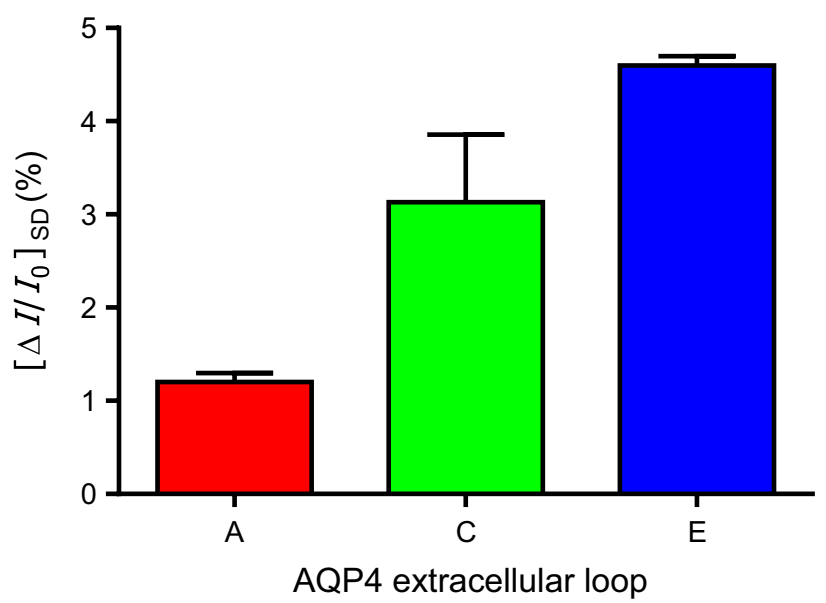

Fig. 5. (a) Real-time response to $1 \mathrm{~g} \mathrm{l}^{-1}$ of AQP4 antibody in $1 / 10^{5}$-diluted human serum. (b) Quantitative comparison of conductance change among AQP4 extracellular loop A (red line), C (green line) and E (blue line). Error bars, s.e.m. three replicates. (For interpretation of the references to color in this figure legend, the reader is referred to the web version of this article.)

Lim, J.H., Park, J., Oh, E.H., Ko, H.J., Hong, S., Park, T.H., 2014. Adv. Healthc. Mater. 3, 360-366.

Marnetto, F., Hellias, B., Granieri, L., Frau, J., Patanella, A.K., Nytrova, P., Sala, A. Capobianco, M., Gilli, F., Bertolotto, A., 2009. J. Neuroimmunol. 217, 74-79.

McKeon, A., Fryer, J.P., Apiwattanakul, M., Lennon, V.A., Hinson, S.R., Kryzer, T.J., Lucchinetti, C.F., Weinshenker, B.G., Wingerchuk, D.M., Shuster, E.A., Pittock, S. J., 2009. Arch. Neurol. 66, 1134-1138.

Park, J., Lim, J.H., Jin, H.J., Namgung, S., Lee, S.H., Park, T.H., Hong, S., 2012. Analyst 137, 3249-3254.

Park, E.J., Park, J., Song, H.S., Kim, S.J., Jung, K.C., Kim, S., Cho, D., Kim, D., Park, K.S., Hong, S., 2014. Biosens. Bioelectron. 61, 140-146.

Paul, F., Jarius, S., Aktas, O., Bluthner, M., Bauer, O., Appelhans, H., Franciotta, D., Bergamaschi, R., Littleton, E., Palace, J., Seelig, H.P., Hohlfeld, R., Vincent, A., Zipp, F., 2007. PLoS Med. 4, e133.

Pisani, F., Mastrototaro, M., Rossi, A., Nicchia, G.P., Tortorella, C., Ruggieri, M., Trojano, M., Frigeri, A., Svelto, M., 2011. J. Biol. Chem. 286, 9216-9224.

Poenitzsch, V.Z., Winters, D.C., Xie, H., Dieckmann, G.R., Dalton, A.B., Musselman, I. H., 2007. J. Am. Chem. Soc. 129, 14724-14732.

Song, H.S., Jin, H.J., Ahn, S.R., Kim, D., Lee, S.H., Kim, U.K., Simons, C.T., Hong, S., Park, T.H., 2014. ACS Nano 8, 9781-9789.

Takahashi, T., Fujihara, K., Nakashima, I., Misu, T., Miyazawa, I., Nakamura, M., Watanabe, S., Shiga, Y., Kanaoka, C., Fujimori, J., Sato, S., Itoyama, Y., 2007. Brain $130,1235-1243$.

Waters, P., Jarius, S., Littleton, E., Leite, M.I., Jacob, S., Gray, B., Geraldes, R., Vale, T., Jacob, A., Palace, J., Maxwell, S., Beeson, D., Vincent, A., 2008. Arch. Neurol. 65, 913-919.

Wingerchuk, D.M., Hogancamp, W.F., O’Brien, P.C., Weinshenker, B.G., 1999. Neurology 53, 1107-1114.

Wingerchuk, D.M., Lennon, V.A., Pittock, S.J., Lucchinetti, C.F., Weinshenker, B.G., 2006. Neurology 66, 14851489. 